\title{
I GUARDIANI DEI DIRITTI DELLA NATURA E DELLE SUE ENTITÀ
}

di Livio Perra

\section{Introduzione}

Nell'ultimo decennio si sta assistendo all'emergere in vari luoghi del pianeta di nuove impostazioni giuridiche che, al fine di assicurare una tutela ambientale più efficace, cambiano il modo di scrivere le norme, di interpretarle e, più in generale, mettono in discussione la classica costruzione del diritto di matrice antropocentrica. In questi ordinamenti giuridici l'accento non è più posto solo sull'essere umano $\mathrm{e}$, di conseguenza, il diritto non viene più costruito esclusivamente attorno all'uomo o in funzione di esso. In tal modo, la natura o alcune entità naturali divengono soggetti o persone con veri e propri diritti.

L'egemonia dell'essere umano rispetto ad altre entità naturali svanisce pian piano, o meglio sfuma nell'indistinto, nei sistemi di protezione che prevedono una ritrovata nobiltà per la natura e le sue entità. L'uomo viene concepito come parte della natura, ora emancipata dal mondo delle res, ed inserito in un sistema più ampio dove con essa contribuisce a formare un tutto vivo. Questi sistemi sono nati come risposta alla problematica ambientale e costituiscono una possibile soluzione. In questo decennio giungono buoni segnali da queste nuove prospettive giuridiche e si assiste alla crescente diffusione di simili impostazioni in vari Paesi.

L'affermazione dei diritti attribuiti ad entità naturali non umane costituisce sicuramente un fatto che desta stupore e affascina i giuristi di tutto il mondo, ma occorre comprendere come questi diritti siano intesi ed in quali categorie possono essere ricompresi. Altro aspetto che ne-

Dipartimento di Storia, Scienze dell’Uomo e della Formazione, Università di Sassari. 
cessita di un approfondito esame è quello relativo al rapporto che intercorre tra i diritti della natura e delle sue entità e l'essere umano. Sarà, perciò, necessario indagare il ruolo a cui l'essere umano è chiamato in questi nuovi sistemi di protezione ambientale al fine di tutelare i diritti della natura e delle sue entità.

In questo lavoro l'attenzione sarà, pertanto, dedicata in primis all'analisi degli aspetti relativi ai nuovi diritti della natura e delle sue entità, la cui trattazione si rende necessaria al fine di comprendere in secundis il ruolo dell'uomo dinnanzi alle esigenze di tutela di tali diritti.

\section{I diritti della natura ed i diritti delle entità naturali}

Questo paragrafo, è dedicato all'analisi dei diritti della natura e dei diritti delle entità naturali. In particolare, occorre osservare quali nuovi diritti sono previsti nelle varie esperienze giuridiche che hanno optato per una scelta non antropocentrica in materia ambientale. In seguito, sullo scenario di questa osservazione sarà innescata la riflessione necessaria all'inquadramento in opportune categorie dei medesimi diritti, grazie all'esame delle principali teorie dottrinali e degli orientamenti giurisprudenziali che si occupano di tale aspetto.

Osservando le costituzioni, le leggi e le sentenze che si occupano dei diritti della natura, si può rilevare la previsione di diritti attribuiti ad entità naturali non umane.

In alcuni esempi vi sono chiari riferimenti a due tipologie di diritti: $i$ diritti relativi all'esistenza e quelli relativi alla restaurazione, al ripristino, nel caso in cui si siano verificati dei danni ambientali. Al fine di comprendere l'individuazione di queste due tipologie di diritti, bisogna osservare da vicino i testi dove sono previsti e affermati gli stessi diritti.

Il primo esempio della previsione delle due tipologie di diritti della natura è presente nella Costituzione dell'Ecuador del 2008'1. La

1 Si vedano a tal proposito: L. PERra, La natura e $i$ suoi diritti, in "Nomos: le attualità nel diritto", n. 2, 2018, p. 8; L. Perra, Etnodesarrollo jurídico y protección del medio ambiente, in "Veredas do Direito: Direito Ambiental e Desenvolvimento Sustentável", n. 34, 2019, p. 79; L. Perra, Tradition Can Save the Future of Nature: Biocentric View of Law, in E.G. Dobbins, M.L. Piga, L. Manca (a cura), "Environment, Social Justice, and the Media in the Age of the Anthropocene", Lanham, MD, Lexington Books, 2020, p. 344. 
natura è riconosciuta "soggetto di quei diritti che le riconosce la Costituzione"2 nel secondo comma dell'articolo 10. In tale Costituzione i diritti relativi all'esistenza sono previsti nell'articolo 71, secondo il quale: "la natura o Pacha Mama, dove si riproduce e realizza la vita, ha diritto a che si rispetti integralmente la sua esistenza e al mantenimento e rigenerazione dei suoi cicli vitali, struttura, funzioni e processi evolutivi"3.

Nell'articolo 72 della medesima Costituzione è affermato il diritto della natura alla restaurazione. In particolare, in questo articolo si legge che: "la natura ha diritto alla restaurazione. Questa restaurazione sarà indipendente dall'obbligo che hanno lo Stato e le persone fisiche o giuridiche di indennizzare gli individui e le collettività che dipendono dai sistemi naturali colpiti"4.

Un secondo esempio può essere rintracciato nella Legge n. 71 del 2010 della Bolivia [Ley de Derechos de la Madre Tierra], la quale fissa nell'articolo 5 il carattere giuridico della Madre Terra, definendola "soggetto collettivo di interesse pubblico"s. Nell'articolo 7, accanto ai diritti relativi all'esistenza è contemplato il diritto della Madre Terra alla restaurazione. Nello specifico, in tale articolo, sono sanciti i diritti "alla vita"6, "alla diversità della vita", "all'acqua", "all'aria pulita"9, "all'equilibrio"10, "alla restaurazione"11, "a vivere libera dalla contaminazione" 12 . Vi è, inoltre, uno spazio lasciato aperto dall'articolo 5 della stessa legge, il quale prevede che al di fuori di tale legge potranno esistere altri diritti della Madre Terra.

\footnotetext{
2 "Sujeto de aquellos derechos que le reconozca la Constitución".

3 "La naturaleza o Pacha Mama, donde se reproduce y realiza la vida, tiene derecho a que se respete integralmente su existencia y el mantenimiento y regeneración de sus ciclos vitales, estructura, funciones y procesos evolutivos".

4 "La naturaleza tiene derecho a la restauración. Esta restauración será independiente de la obligación que tienen el Estado y las personas naturales o jurídicas de indemnizar a los individuos y colectivos que dependan de los sistemas naturales afectados".

5 "Sujeto colectivo de interés público".

6 "A la vida".

7 "A la diversidad de la vida".

8 "Al agua".

9 "Al aire limpio"

10 "Al equilibro".

11 "A la restauración".

12 "A vivir libre de contaminación".
} 
Un terzo esempio è offerto dalla sentenza T-622/16 della Corte Costituzionale della Colombia. Tale sentenza dichiara il fiume Atrato "soggetto dei diritti che implicano la sua protezione, conservazione, mantenimento e $[\ldots]$ restaurazione" 13 .

In Nuova Zelanda, facendo seguito ad accordi intervenuti tra il popolo Whanganui River Iwi ed il governo e altri atti ${ }^{14}$, si è addivenuti al riconoscimento del fiume Whanganui River [Te Awa Tupua] come persona giuridica. A questo proposito è utile soffermarsi sulla lettura del Te Awa Tupua (Whanganui River Claims Settlement) Act 2017, nel quale si legge, nella Subpart 2 della Part 2, che: "il Te Awa Tupua è una persona giuridica e ha tutti i diritti, i poteri, i doveri e le responsabilità di una persona giuridica"15.

In India, la High Court of Uttarakhand at Nainital afferma che: " $\mathrm{i}$ fiumi Ganga e Yamuna, tutti i loro affluenti, flussi, ogni acqua naturale che scorre con flusso continuo o intermittente di questi fiumi, sono dichiarati come persone giuridiche/legali/entità viventi aventi lo status di persona giuridica con tutti i diritti, i doveri e le responsabilità corrispondenti [a quelli] di una persona vivente al fine di preservare e conservare il fiume Ganga e Yamuna"16.

In questo caso la Corte della città di Nainital nello Stato indiano di Uttarakhand identifica i fiumi Ganga e Yamuna nella loro totalità, compresi anche i loro affluenti, come persone giuridiche particolari

${ }^{13}$ Corte Constitucional de Colombia, Sentencia T-622/16, punto 9.25., p. 140; punto 9.32., p. 145: "sujeto de derechos que implican su protección, conservación, mantenimiento y $[\ldots]$ restauración".

${ }^{14} \mathrm{Si}$ pensi ad esempio all'accordo stipulato il 5 agosto 2014 tra i rappresentanti del popolo Whanganui River Iwi ed il governo neozelandese. Si vedano a tal proposito: Whanganu Iwi And The Crown, Ruruku Whakatupua - Te Mana o Te Iwi o Whanganui, 5 August 2014; A. SALMOND, Tears of Rangi: Water, power, and people in New Zealand, in "Hau: Journal of Ethnographic Theory", n. 3, 2014, pp. 285-286.

15 "Te Awa Tupua is a legal person and has all the rights, powers, duties, and liabilities of a legal person".

${ }^{16}$ High Court of Uttarakhand at Nainital, Mohd. Salim v. State of Uttarakhand \& others, Writ Petition (PIL) No. 126 of 2014. March 20, 2017, punto 19, p. 11: "The Rivers Ganga and Yamuna, all their tributaries, streams, every natural water flowing with flow continuously or intermittently of these rivers, are declared as juristic/legal persons/living entities having the status of a legal person with all corresponding rights, duties and liabilities of a living person in order to preserve and conserve river Ganga and Yamuna". 
in quanto sono ritenute entità viventi e sono loro riconosciuti i diritti, i doveri e le responsabilità propri delle "persone viventi".

In Bangladesh, la High Court Division of the Supreme Court nel 2019 giudica il caso relativo al fiume Turag (Writ Petition No. 13989 of 2016), emettendo un'articolata sentenza. La Corte prevede 17 istruzioni necessarie per proteggere i fiumi. Il fiume Turag è riconosciuto come persona giuridica/entità giuridica/entità vivente $\mathrm{e}$ tale status è esteso a tutti i fiumi che scorrono nel Paese o lo attraversano. In particolare, nella seconda istruzione essa afferma che: "il fiume Turag è dichiarato come persona giuridica (legal person)/ entità giuridica (legal entity)/ entità vivente (living entity).Tutti i fiumi che scorrono all'interno e attraverso il Bangladesh avranno lo stesso status" 17 .

Dopo aver osservato gli esempi del riconoscimento della natura e delle sue entità come soggetti o persone e l'attribuzione ad esse dei loro diritti, occorre riflettere se sia possibile ricondurre questi ultimi a categorie già presenti nello scenario giuridico. A tal fine, è utile richiamare le classificazioni che sono state effettuate dei diritti della natura e delle sue entità dalla dottrina e dalla giurisprudenza.

Per prima cosa, occorre evidenziare che i diritti della natura e delle sue entità sono un inedito. Infatti, è la prima volta che il mondo giuridico si trova di fronte ad una categoria di diritti attribuiti a soggetti della natura diversi dall'essere umano.

La questione che deve essere affrontata per ricondurre a categoria questa nuova tipologia di diritti risiede nel comprendere se si tratti di diritti totalmente autonomi ed indipendenti da quelli degli esseri umani o, invece, se siano da essi derivati.

Sul punto non vi è un quadro teorico univoco ma lo scenario teorico può essere ricondotto a due concezioni.

La prima concezione vede nei diritti della natura e delle sue entità una categoria del tutto autonoma e indipendente.

Alberto Acosta per ricondurre i diritti della natura ad una categoria, trova nella volontà del popolo ecuadoriano espressa nella Costituzione dell'Ecuador, attraverso l'attività dei membri dell'assemblea

${ }^{17}$ High Court Division of The Supreme Court of Bangladesh, Human Rights and Peace for Bangladesh Vs Bangladesh and others, Writ Petition 13989/2016, istruzione 2, p. 278. La traduzione dal bengalese è dell'autore. 
costituente, il punto di partenza su cui sviluppa la propria riflessione. Questa stessa volontà si è manifestata con una amplissima maggioranza in sede di approvazione referendaria della Costituzione il 28 settembre 2008. La Costituzione dell'Ecuador esprime due categorie distinte e differenti di diritti: i diritti ecologici ed i diritti ambientali ${ }^{18}$.

I diritti ecologici sono posti a tutela dei cicli vitali e dei processi evolutivi, della sopravvivenza delle specie e degli ecosistemi. Si tratta di una tipologia di diritti autonoma rispetto ai diritti degli esseri umani. La violazione di tali diritti ricade nel campo della giustizia ecologica, la quale è dedicata totalmente alla natura e non si occupa del risarcimento spettante agli esseri umani in conseguenza di danni ambientali 19 .

I diritti ambientali, "che sorgono dai diritti umani" 20 , sono strettamente connessi all'essere umano. La giustizia ambientale è dedicata alle persone ${ }^{21}$.

Concordemente a quanto sottolineato da Eduardo Gudynas ${ }^{22}$, nella Costituzione dell'Ecuador si può rilevare un doppio binario in materia ambientale: negli articoli 71 e 72 sono previsti i diritti della natura, mentre nell'articolo 14 è riconosciuto il diritto degli individui a vivere in un ambiente sano ed ecologicamente equilibrato. In particolare, tale diritto è un classico diritto degli esseri umani, che viene ricondotto alla categoria dei diritti umani di terza generazione ${ }^{23}$.

${ }^{18} \mathrm{Si}$ veda a tal proposito: A. Acosta, Los Derechos de la Naturaleza. Una lectura sobre el derecho a la existencia, in A. AcostA, E. MARTínez (a cura), "La naturaleza con derechos. De la filosofía a la política", Quito, Abya-Yala, 2011, pp. 354-355.

${ }^{19} \mathrm{Si}$ veda a tal proposito: A. Acosta, Los Derechos de la Naturaleza. Una lectura sobre el derecho a la existencia, cit., p. 355.

20 A. Acosta, Los Derechos de la Naturaleza. Una lectura sobre el derecho a la existencia, cit., p. 355: "que surgen desde los Derechos Humanos".

${ }^{21} \mathrm{Si}$ veda a tal proposito: A. Acosta, Los Derechos de la Naturaleza. Una lectura sobre el derecho a la existencia, cit., p. 355.

${ }^{22} \mathrm{Si}$ veda a tal proposito: E. GUDYNAS, Desarrollo, derechos de la naturaleza y Buen vivir después de Montecristi, in G. WeBER (a cura), "Debates sobre cooperación y modelos de desarrollo. Perspectivas desde la sociedad civil en el Ecuador", Quito, Centro de Investigaciones CIUDAD y Observatorio de la Cooperación al Desarrollo, 2011, pp. 87-88.

${ }^{23} \mathrm{Si}$ veda a tal proposito: E. GUDYNAS, Desarrollo, derechos de la naturaleza y Buen vivir después de Montecristi, cit., p. 87. 
Discorso analogo può essere fatto riguardo al fenomeno osservabile in Bolivia. Nello specifico, all' articolo 33 della Costituzione è previsto il diritto degli individui ad un ambiente sano, protetto ed equilibrato. Vi sono chiari riferimenti in materia ambientale ai diritti civili classici nella Costituzione boliviana ${ }^{24}$. Il trionfo del secondo binario è rappresentato nell'articolata legge dedicata ai diritti della Madre Terra [Ley de Derechos de la Madre Tierra] ed in altre leggi dello Stato. Pertanto, anche nella esperienza giuridica boliviana può essere tracciata una netta distinzione tra i diritti ecologici, riservati alla natura, e i diritti ambientali, dedicati al diritto a vivere in un ambiente sano.

Un'altra impostazione è quella che vede nel riconoscimento della natura e delle sue entità come soggetti o persone e nell'affermazione dei loro diritti, l'espressione o il risultato dell'emergere dei diritti bioculturali. Al fine di comprendere questa lettura del fenomeno, è necessario esaminare per prima cosa la definizione di diritti bioculturali.

I diritti bioculturali sono un gruppo di diritti collettivi basati sul ruolo di stewardship proprio della tradizione e della cultura di alcune comunità locali e gruppi indigeni ${ }^{25}$. Tali diritti sono, cioè, quelli legati a doppio filo con la cultura e la tradizione dei popoli che vivono nei propri territori e che garantiscono le forme tradizionali di amministrazione del proprio ambiente secondo la propria cultura. Questi diritti sono rivendicati da comunità locali e popoli indigeni in vari luoghi del pianeta.

La sentenza T-622/16 della Corte Costituzionale della Colombia afferma a tal riguardo che: "a livello internazionale [...] si è sviluppato un nuovo approccio giuridico denominato diritti bioculturali, la cui premessa centrale è la relazione di profonda unità e interdipendenza tra natura e specie umana, e che ha come conseguenza una nuova comprensione socio-giuridica nella quale la natura e il suo ambiente devono essere presi sul serio e con pienezza di diritti”26.

\footnotetext{
${ }^{24}$ Si veda a tal proposito: E. GudynAS, Desarrollo, derechos de la naturaleza y Buen vivir después de Montecristi, cit., p. 87.

${ }^{25}$ Cfr. G. ZANETTI, Diritti bioculturali: percorrendo la strada che separa diverse tradizioni, in "Diritto \& Questioni Pubbliche", n. 1, 2019, p. 269. Si veda a tal proposito anche: K.S. BAVIKATTE, T. BENNETT, Community stewardship: the foundation of biocultural rights, in "Journal of Human Rights and the Environment", n. 1, 2015, pp. 7-9.

${ }^{26}$ Corte Constitucional De Colombia, Sentencia T-622/16, punto 9.28., pp. 142143: "a nivel internacional [...] se ha venido desarrollando un nuevo enfoque jurídico denominado derechos bioculturales, cuya premisa central es la relación de profunda
} 
Secondo la Corte Costituzionale della Colombia si prevede una nuova giustizia che considera la natura e la relazione che con essa ha l'essere umano. La giustizia nei confronti della natura va oltre la dimensione umana e "deve permettere che la natura possa essere soggetto di diritti" 27 .

Seguendo questa ricostruzione operata dalla Corte Costituzionale colombiana si trova un fondamento per i diritti del fiume Atrato, che può essere esteso alla nuova tipologia dei diritti della natura. I diritti della natura e delle sue entità sarebbero, dunque, il frutto di una nuova armonia, di una nuova sensibilità e giustizia ambientale che ha visto il proprio emergere grazie all'affermazione dei diritti bioculturali. In questo modo, si può ritenere che i diritti della natura e delle sue entità abbiano trovato una porta aperta nel sentiero tracciato dai diritti bioculturali che aprivano una breccia nell'approccio giuridico di tipo antropocentrico per giungere ad una giustizia concepita in un'ottica più larga rispetto alla sola dimensione umana.

\section{L'essere umano ed il suo ruolo di guardiano}

In questo paragrafo, si occupa di individuare il ruolo dell'essere umano nel quadro dei diritti della natura e delle sue entità, cioè sottolinea il ruolo che l'uomo esercita nell' ambito che riguarda la tutela dei diritti della natura e delle sue entità.

I Paesi che hanno optato per il riconoscimento dei diritti della natura e delle sue entità si sono trovati a dover fronteggiare una questione di non poco conto. Se da una parte la natura e le entità naturali possono esercitare i propri diritti all'esistenza, dall'altra vi è una difficoltà consistente nel fatto che la natura e le entità naturali non possono attivare di per se stesse i meccanismi per tutelare i propri diritti di fronte agli eventuali attacchi esterni o esercitare le azioni necessarie per ottenere la restaurazione o il ripristino nei casi in cui si veri-

unidad e interdependencia entre naturaleza y especie humana, y que tiene como consecuencia un nuevo entendimiento socio-jurídico en el que la naturaleza y su entorno deben ser tomados en serio y con plenitud de derechos".

${ }^{27}$ Corte Constitucional de Colombia, Sentencia T-622/16, punto 9.31., p. 144: "debe permitir que la naturaleza pueda ser sujeto de derechos". 
fichino danni. Questo fatto non svilisce il riconoscimento della natura e delle sue entità come soggetti o persone. Tutti gli ordinamenti giuridici al loro interno conoscono strumenti atti a sopperire all'incapacità di determinati soggetti di poter provvedere alla cura dei propri interessi. Proprio in queste forme è stata ricercata la soluzione a tale questione dai Paesi che hanno previsto forme di tutela o di rappresentanza ${ }^{28}$. Queste nuove entità giuridiche curano i propri interessi attraverso gli esseri umani, i quali esercitano la tutela o la rappresentanza o forme analoghe in base a quanto previsto sul tema nel proprio Paese. In tal senso, si possono raggruppare tutte queste forme in cui si esplica l'intervento umano in un'unica macrocategoria scegliendo di definire come guardiani dei diritti della natura e delle sue entità gli esseri umani che si occupano di far fronte all'esigenza di curare gli interessi della natura e delle entità naturali.

In una visione quasi favolistica, il ruolo degli esseri umani nella difesa della natura e delle sue entità è espresso nella cultura del popolo Whanganui River Iwi. Tale popolo ed il fiume Te Awa Tupua possiederebbero due antenati in comune: Paerangi e Ruatipua ${ }^{29}$. Questa credenza testimonia il grande legame che questo popolo ha con il fiume. Questa connessione genera "il desiderio dei Whanganui Iwi di prendersi cura, proteggere, gestire e utilizzare il fiume Whanganui attraverso il kawa e il tikanga ${ }^{30}$ conservati dai discendenti di Ruatipua e Paerangi" 31 .

28 Alcuni autori, come ad esempio R. ÁvILA SANTAMARÍA, El derecho de la naturaleza: fundamentos, in A. Acosta, E. MARTínez (a cura), "La naturaleza con derechos. De la filosofía a la política", Quito, Abya-Yala, 2011, pp. 197-202, analizzano la questione relativa all'applicazione di tali strumenti in favore della natura.

${ }^{29} \mathrm{Si}$ veda a tal proposito: E.C. HsIAO, Whanganui River Agreement - Indigenous Rights and Rights of Nature, in "Environmental Policy and Law", n. 6, 2012, p. 371.

${ }^{30} \mathrm{Il}$ termine ' $k a w a$ ' indica tutti quei rituali che scandiscono la vita dei popoli Maori. Con il termine 'tikanga' si indicano le consuetudini, le regole o i metodi del popolo Maori che contribuiscono ad ordinare la vita della società. Si veda a tal proposito: P.F. MAJUREY, H. Atkins, V. Morrison, T. Hovell, Mäori Values Supplement, New Zealand Ministry of the Environment, 2010, p. 274. Cfr. H.W. Williams, Dictionary of the Mäori Language, Wellington, GP Publications, 1971, pp. 416-417; H.M. MEAD, Tikanga Māori: Living by Māori Values, Wellington, Huia Publishers, 2003, p. 12.

31 Whanganul Iwi and The Crown, Tütohu Whakatupua, 30 August 2012, paragrafo 1.3.: "The desire of Whanganui Iwi to care, protect, manage and use the Whanganui River through the kawa and tikanga maintained by the descendants of Ruatipua and Paerangi". Si veda a tal proposito anche: E.C. HsIAO, Whanganui River Agreement - Indigenous Rights and Rights of Nature, cit., p. 371. 
Ora, al fine di comprendere quali siano le forme prescelte nelle singole esperienze dei Paesi che hanno optato per l'attribuzione alla natura e alle sue entità di veri e propri diritti, occorre analizzare i testi normativi e la giurisprudenza che hanno trattato questi argomenti.

In Ecuador, nel secondo comma dell'articolo 71 è previsto che: “ogni persona, comunità, popolo o nazione potrà esigere dall'autorità pubblica il compimento dei diritti della natura" 32 .

Si tratta in questo caso di una ampia legittimazione a richiedere il compimento dei diritti della natura.

In Bolivia, l'articolo 34 della Costituzione stabilisce che: "qualunque persona, a titolo individuale o in rappresentanza di una collettività, ha la facoltà di esercitare le azioni giuridiche in difesa del diritto ambientale, senza pregiudizio per gli obblighi delle istituzioni pubbliche di agire d'ufficio di fronte agli attacchi contro l'ambiente"33.

È necessario, poi, volgere lo sguardo all'articolo 39 della legge boliviana n. 300 del 2012 [Ley Marco de la Madre Tierra y Desarrollo Integral para Vivir Bien]. Tale articolo prevede che le autorità pubbliche in base alle loro competenze, il Ministerio Público, la Defensoría de la Madre Tierra, il Tribunale Agroambientale, le persone direttamente colpite devono proporre le istanze amministrative e giurisdizionali per difendere e garantire i diritti della Madre Terra, nel quadro dello sviluppo integrale per il Vivere Bene [Vivir Bien]. Inoltre, le persone individuali o collettive hanno il dovere di denunciare le violazioni dei diritti della Madre Terra di cui abbiano conoscenza.

In Colombia, la sentenza T-622/16 della Corte Costituzionale stabilisce che dovrà essere formata una commissione di guardiani del fiume Atrato, al fine di proteggere, recuperare e preservare lo stesso fiume. Questa commissione sarà costituita dai guardiani designati e da un gruppo di consulenti. All'interno di questa commissione vi saranno come guardiani un membro delle comunità ed un delegato dal governo colombiano ${ }^{34}$.

32 "Toda persona, comunidad, pueblo o nacionalidad podrá exigir a la autoridad pública el cumplimiento de los derechos de la naturaleza".

33 "Cualquier persona, a título individual o en representación de una colectividad, está facultada para ejercitar las acciones legales en defensa del derecho al medio ambiente, sin perjuicio de la obligación de las instituciones públicas de actuar de oficio frente a los atentados contra el medio ambiente".

${ }^{34} \mathrm{Si}$ veda a tal proposito: Corte Constitucional de Colombia, Sentencia T-622/16, pp. $145 ; 159 ; 164$. 
In Nuova Zelanda, nel Te Awa Tupua (Whanganui River Claims Settlement) Act 2017 viene istituito il Te Pou Tupua, definito come "volto umano del Te Awa Tupua"35. Il Te Pou Tupua agisce in nome del medesimo fiume e possiede i poteri per esercitare le proprie funzioni. Per quanto concerne la composizione, esso è formato da due membri. Questi membri vengono nominati rispettivamente dagli Iwi e dal Minister for Treaty of Waitangi Negotiations dopo aver consultato il Minister for Māori Development, il Minister of Conservation ed il Minister for the Environment, in nome della Corona. Vi sono leggere differenze per le nomine successive, in quanto saranno effettuate dal Minister for the Environment, che si consulterà con il Minister for Māori Development, il Minister of Conservation. Sarà, inoltre, consultato ogni altro Minister nel caso in cui ve ne sia necessità per conseguire gli scopi del Te Poи Tuриа. Per essere nominati occorre possedere adeguati requisiti utili per conseguire gli scopi ed esercitare le funzioni del Te Pou Tupua: abilità, conoscenza ed esperienza. Chi nomina l'altro membro riceve il nome del soggetto proposto e valuterà se sussistono i necessari requisiti fornendo un suo parere sulla proposta. In seguito, la nomina dei due membri avverrà congiuntamente ${ }^{36}$.

In India, la High Court of Uttarakhand at Nainital prevede che: "il Director Namami Gange, il Chief Secretary of the State of Uttarakhand e l'Advocate General of the State of Uttarakhand sono dichiarate le persone che in loco parentis provvederanno a proteggere, conservare e preservare i fiumi Ganga e Yamuna ed i loro affluenti" 37.

Essi difenderanno lo status dei fiumi Gange e Yamuna e ne promuoveranno la salute ed il benessere ${ }^{38}$. Inoltre, la Corte stabilisce che l'avvocato generale nei procedimenti giudiziari per la tutela degli interessi dei fiumi Gange e Yamuna rappresenterà i due fiumi ${ }^{39}$.

35 "Human face of Te Awa Tupua".

${ }^{36}$ Si veda a tal proposito: Te Awa Tupua (Whanganui River Claims Settlement) Act 2017.

${ }^{37}$ High Court of Uttarakhand at Nainital, Mohd. Salim v. State of Uttarakhand \& others, cit., punto 19, pp. 11-12: "The Director NAMAMI Gange, the Chief Secretary of the State of Uttarakhand and the Advocate General of the State of Uttarakhand are hereby declared persons in loco parentis as the human face to protect, conserve and preserve Rivers Ganga and Yamuna and their tributaries".

${ }^{38} \mathrm{Si}$ veda a tal proposito: High Court of UtTarakhand at Nainital, Mohd. Salim v. State of Uttarakhand \& others, cit., punto 19, p. 12.

${ }^{39} \mathrm{Si}$ veda a tal proposito: High Court of UtTarakhand at Nainital, Mohd. Salim v. State of Uttarakhand \& others, cit., punto 20, p. 12. 
In Bangladesh, la High Court Division of the Supreme Court of Bangladesh stabilisce che la Commissione Nazionale per la Protezione dei Fiumi, in loco parentis, si occuperà della protezione, della conservazione e dello sviluppo e proteggerà dall'inquinamento e dalla occupazione illegale il fiume Turag e tutti i fiumi del Paese ${ }^{40}$.

Dall'osservazione dei Paesi dove si è scelto di riconoscere la natura o le sue entità come soggetti o persone e la conseguente attribuzione di veri e propri diritti, emerge il fatto che quando ci si trova dinnanzi all'incapacità della natura e delle sue entità di curare i propri interessi, interviene l'essere umano. Al pari di quanto avviene per i soggetti incapaci, gli ordinamenti predispongono strumenti adatti per lo svolgimento delle necessarie attività. Così saranno gli uomini ad agire nelle opportune sedi per tutelare i diritti della natura e delle sue entità. In alcuni casi si tratta di una legittimazione ampia attribuita a tutti gli esseri umani, in altri casi questa incombenza è attribuita in maniera specifica ad enti o commissioni esistenti o appositamente create. Da un punto di vista concettuale questa soluzione non contrasta con le logiche degli ordinamenti giuridici, in quanto erano già presenti situazioni dove all'incapacità di un soggetto si sopperiva con appositi strumenti.

\section{Conclusioni}

Nell'ultimo decennio in vari Paesi emerge il riconoscimento della natura e delle sue entità come soggetti o persone e conseguentemente vengono ad esse attribuiti veri e propri diritti. Si tratta di un cambio di prospettiva rispetto alla consueta visione antropocentrica, in quanto il mondo giuridico non viene più pensato solo ed esclusivamente in funzione dell'essere umano, ma si è dato il via ad una nuova fase del diritto che pone sullo stesso piano di dignità la natura e le sue entità con l'uomo. Concretamente accade che alcuni Stati disegnano i diritti della natura e delle sue entità ed i meccanismi con cui garantirli e tutelarli.

Per prima cosa nel presente lavoro si è voluto volgere lo sguardo verso il riconoscimento della natura e delle sue entità come soggetti o

${ }^{40} \mathrm{Si}$ veda a tal proposito: High Court Division of The Supreme Court of Bangladesh, Human Rights and Peace for Bangladesh Vs Bangladesh and others, cit., istruzione 3, pp. 278-279. 
persone ed i diritti loro attribuiti. Da questa osservazione sono scaturite le riflessioni basate sugli approcci offerti dalla dottrina e dalla giurisprudenza al fine di inquadrare tali diritti sotto una precisa categoria.

In seconda battuta si è voluto verificare se vi fosse un ruolo per l'essere umano che affiancasse questa cornice composta dai nuovi diritti attribuiti ad entità naturali non umane. L'autore ha rilevato come i Paesi, che hanno scelto di innovare in materia ambientale, prevedendo veri e propri diritti in capo alla natura o alle sue componenti, hanno dovuto affrontare la questione relativa alla concreta tutela della nuova tipologia di diritti. La natura e le sue entità non sempre sono capaci di provvedere alla cura dei propri interessi ed in questi casi i Paesi hanno previsto delle forme nelle quali gli uomini possono richiedere la tutela dei diritti, esercitare le necessarie azioni a loro difesa e, più in generale, proteggere l'ambiente. La gamma di soluzioni con cui l'essere umano può sostituirsi alla natura o alle sue entità per provvedere ad ogni incombenza si esplicano sotto varie forme.

L'autore ha denominato gli esseri umani che si muovono in queste multiformi configurazioni guardiani dei diritti della natura e delle sue entità. In alcuni casi vi è una ampia legittimazione concessa indistintamente a tutti gli esseri umani, in altri casi sono designati per tale compito enti o commissioni già esistenti o creati $a d$ hoc. I testi giuridici e giurisprudenziali parlano di tutela o rappresentanza ed in alcuni casi gli enti o le commissioni agiscono in loco parentis al fine di proteggere la natura e le sue entità. In ogni caso, la natura e le sue entità sono i veri titolari dei diritti, ma come se fossero soggetti incapaci che non riescono a curare da sole i propri interessi, trovano nell'essere umano un alleato che si preoccupa delle vicende che le riguardano. Così facendo, questi sistemi assumono una veste di completezza ed i diritti delle entità naturali non umane non rimangono solo sulla carta, ma ricevono una tutela concreta.

\footnotetext{
Abstract - In this article, the author analyses the rights of nature and the guardianship exercised by the human being. For this purpose, he first observes and analyses the rights of nature and its entities. Moreover, the reflection is aimed at finding a category where these rights can be included. From the analysis of these issues, attention shifts to the need to understand the role that human beings play in the framework of the rights of nature and its entities. Na-
}

ture and its entities alone are unable to take care of their interests, in particular to claim respect for their rights and to invoke restoration in the event that damage occurs. Human beings make up for the incapacity of nature or its entities through specific tools. The author analyses the various tools that are used in some Countries, leads them back to a single macro-category and defines men acting for natural entities as guardians of the rights of nature and its entities. 